\title{
Heterodyne Spectroscopy In The Far Infrared
}

\author{
H.P. Röser \\ Max-Planck-Institut für Radioastronomie, \\ Bonn, Germany
}

\begin{abstract}
A high resolution heterodyne spectrometer for the submillimetre/far infrared wavelength range has been developed for measurements in astronomy. Robust equipment intended for airborne operation and capable of detecting very weak signals was required. The heterodyne technique has now been extended to applications in other areas.
\end{abstract}

In the case of astronomical spectroscopy, the wavelength band between 100 and $1000 \mu \mathrm{m}$ remains one of the last sectors of the electromagnetic spectrum that has not been completely opened up. Covering the so-called submillimetre and far infrared (FIR) spectral ranges from 100 to $500 \mu \mathrm{m}$ that have not yet been standardized, the band contains numerous spectral lines of prime significance. Included are those used to study interstellar matter involving low energy rotational transitions of small and light molecules such as hydrides, and the higher transitions of somewhat heavier molecules such as carbon monoxide $(\mathrm{CO})$ in excited rotational states corresponding to elevated temperatures. Important fine structure transitions for neutral oxygen $\left(\mathrm{O}^{\circ}\right)$, ionized carbon $\left(\mathrm{C}^{+}\right)$and nitrogen $\left(\mathrm{N}^{+}\right)$which, in contrast to molecules, do not have transitions in the microwave range are also present.

Observation of these various lines promises to advance the understanding of interstellar chemistry, the energy content of interstellar clouds, and star formation in different regions of our Galaxy. For example, cores comprising clouds of molecules that may have an embedded protostar are generally very opaque, especially at the line centre. This feature means that one is unable to observe low CO transitions (rotational

Dr. H.P. Röser is with the Max-Planck-Institut für Radioastronomie, Auf dem Hugel 69, D-5300 Bonn. He is a principal investigator on KAO in the USA and Chairman of the German committee for the proposed SOFIA airborne observatory. quantum number $J<4$ ) deep within the core. The less opaque cold gas in the foreground does not emit at the higher rotational transitions $(J>6)$ so these transitions can be used to observe the embedded source. In other words, information comes from deeper inside the cloud the higher the $\mathrm{CO}$ transition that is examined. By monitoring the different higher transitions of $\mathrm{CO}$ and observing several warm and hot star formation regions, one hopes to obtain temperature and density as a function of velocity and, in some cases, of position. The data can then be analysed with respect to the global properties of the regions.

\section{Atmospheric Transmission}

The Earth's atmosphere between an observer and an astronomical object along a line of sight acts as a filter which blocks nearly all astronomical signals at adsorption bands involving mainly oxygen, ozone and water vapour. The translucent (permeable) spec- tral ranges between the pressure broadened adsorption lines are also affected. With the exception of some spectral windows down to $350 \mu \mathrm{m}$, where favourable occasions permit measurements of objects at high elevations from ground-based telescopes placed on high altitude mountain tops, the submillimetre wavelength band is only accessible for astronomical observations from above the troposphere. Platforms such as airplanes, balloons and satellites carry relatively small telescopes which, for the wavelengths of interest, imply a low spatial resolution. It is therefore of prime importance to make measurements at very high spectral resolutions in order to distinguish between different components of an object through their different velocities, as manifested by the relative Doppler shifts $(1 \mathrm{~km} / \mathrm{s}$ is equivalent to a frequency shift of $\approx 3 \mathrm{MHz}$ at a wavelength $\lambda$ of $300 \mu \mathrm{m}$ ).

Fig. 1 shows the atmospheric transmission for each of the important mole-

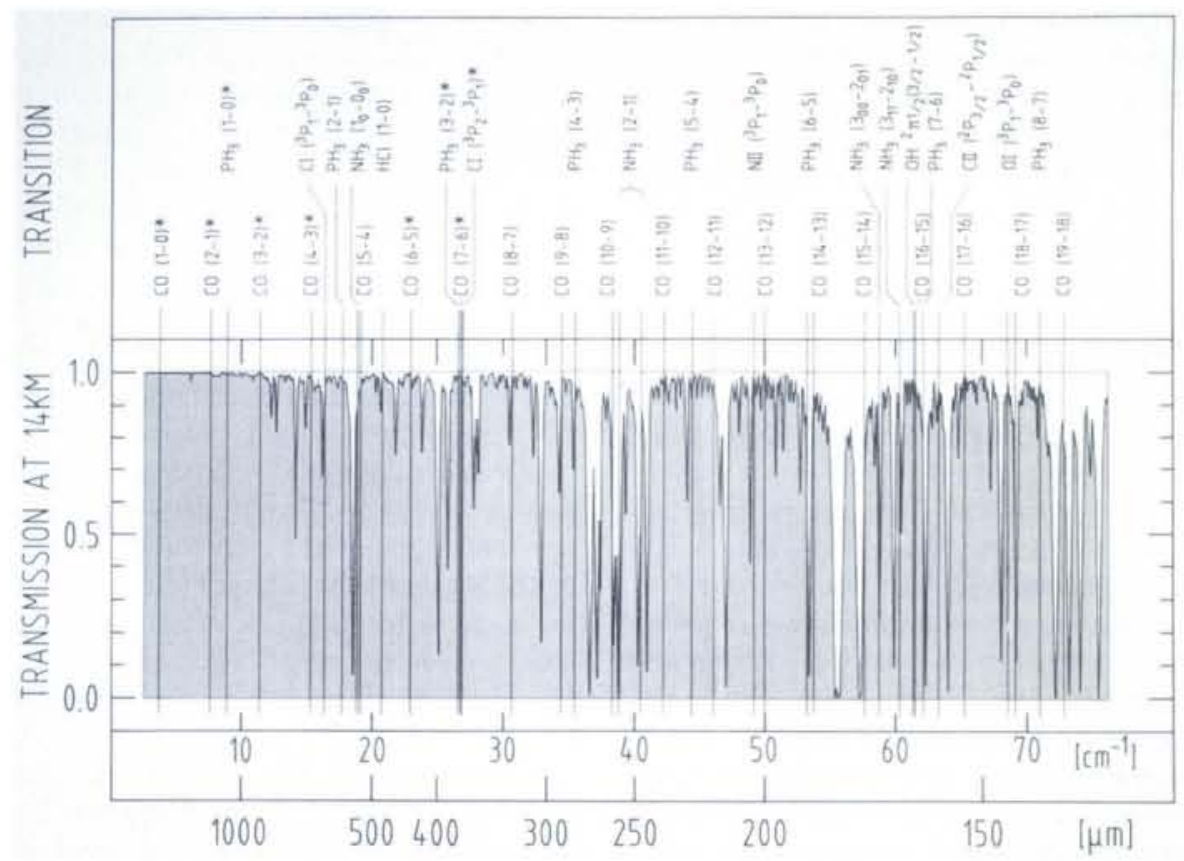

Fig. 1 - Atmospheric transmission at the airborne research operation altitude of $14 \mathrm{~km}$. Values are given for transitions between rotational quantum levels $\mathrm{J}$ of various molecules. The spectral lines marked with an asterisk are observable on the ground. 
cular and atomic transitions used in astronomy and atmospheric physics. The data are for the altitude of $14 \mathrm{~km}$ (45000 feet) used in most airborne research operations. The transitions marked with an asterisk are also observable from the ground.

\section{Kuiper Airborne Observatory}

Astronomers have mostly been allowed a view of the submillimetre and far infrared because of KAO, the Kuiper Airborne Observatory, a national facility in the USA that has been operated by NASA since 1974. The observation platform is a modified C-141A jet transport with a range of $11000 \mathrm{~km}$ and capable of conducting research operations to an altitude of $14 \mathrm{~km}$. KAO's telescope comprises a conventional Cassegrain reflector with a $91.5 \mathrm{~cm}$ aperture that was designed primarily for operations in the $1-500 \mu \mathrm{m}$ range. It views athwartships from an open cavity recessed in the aircraft's fuselage. Although the telescope is rather small for use at long wavelengths, its availability above the troposphere has proved to be of enormous value.

\section{Spectrometers}

FIR and submillimetre spectral lines have interesting velocity structures for speeds ranging from hundreds of $\mathrm{km} / \mathrm{s}$ - as seen in external galaxies and in the galactic centre - to fractions of a $\mathrm{km} / \mathrm{s}$. Structures for the latter have also been observed previously in lines originating in clouds of molecules present in our Galaxy. One therefore needs spectrometers with different resolving powers and total bandwidths depending upon the character of the astronomical problem at hand.

Various types of spectrographic techniques have been developed for KAO but basically only two are in regular use. For very high spectral resolutions $(\Delta v / v$ $\sim 10^{-5}-10^{-7}$ where $\Delta v$ is the half power line width at a frequency $v$ ) there are heterodyne spectrometers: for intermediate resolutions $\left(\Delta v / v>10^{-5}\right)$, FabryPerot or grating spectrometers are the preferred choice. Both types have proved themselves and are capable of further development.

New types of heterodyne receivers based on Schottky diodes with tunable open structure mixers and using lasers as local oscillators (LO) now allow us to cover most of the sub-mm/FIR wavelength range at high resolution and with a large bandwidth using a single instrument. Their application, particularly in airborne astronomy, has led to a large number of new discoveries.

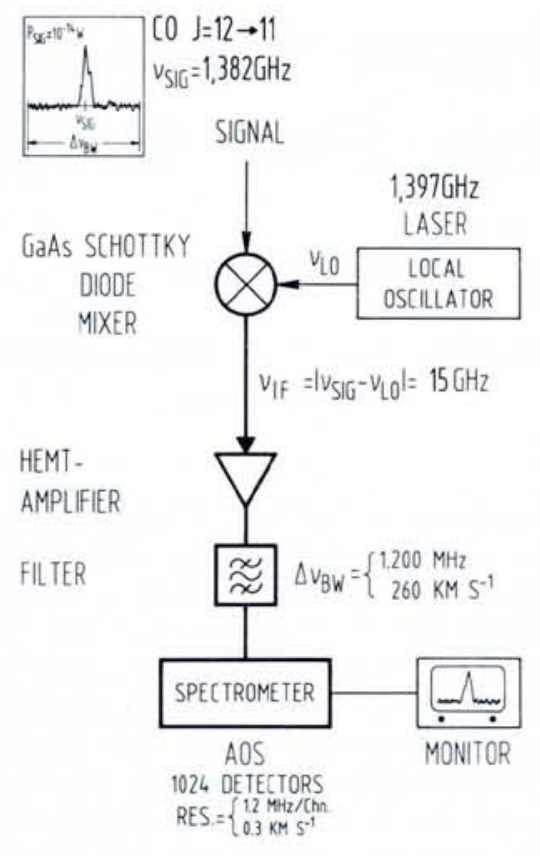

Fig. 2 - Block diagram of the high resolution heterodyne spectrometer. A weak signal with a power $P$ of $10^{-14} \mathrm{~W}$ from the $J=12-11$ transition of carbon monoxide (CO) at a frequency $v_{\text {sig }}=1.382 \mathrm{GHz}$ is mixed in a diode mixer with a local oscillator beam having a similar frequency for conversion into a signal with an intermdiate frequency (IF). After amplification and filtering, the signal profile is measured using an acousto-optic spectrometer (AOS) with 1024 detectors.

\section{Sub-mm/FIR Heterodyne Techniques}

The heterodyne technique is wellknown in radio communication as the "superheterodyne" receiver that is used commercially for the short to long wavelength range with both frequency and amplitude modulation. The primary function of a heterodyne receiver with a diode mixer is to translate a signal at one frequency to another with a different frequency which can be amplified or processed more efficiently. The ability to shift a signal in frequency with minimal added noise or distortion is important because the properties of amplifiers, filters and detectors depend upon the frequency. In order for these devices to function optimally it is often necessary to shift the signal to suitable frequencies. One of the major advantages of the diode mixer is that it can usually be used at frequencies where nothing else will work, and this is especially the case at sub-mm/FIR wavelengths $(<500 \mu \mathrm{m})$.

As an example, Fig. 2 illustrates the signal processing path for a high resolution heterodyne spectrometer used in the detection of the $\mathrm{CO}$ line with a rotational quantum number $J=12-11$ at $217 \mu \mathrm{m}$ : a very weak signal on the order of $10^{-14} \mathrm{~W}$ can be detected and the line width resolved. The incoming signal is mixed in a nonlinear resistance (diode mixer) with a local oscillator beam having a frequency close to the signal frequency. All the signal information is transferred down to the microwave range where low noise amplifiers and suitable filters exist. A microwave spectrometer generates the line profile which is stored and displayed on a personal computer.

\section{Diode mixer}

GaAs Schottky barrier diodes are the mixer element of choice as they are the only ones that cover the sub-mm and FIR ranges. But a few research laboratories fabricate diodes optimized for the sub-mm regime, and steady improvements in performance are being realized by determining the optimum design for a specific frequency range and operating temperature. This entails consideration of the impurity profile and device geometry, as well as efforts to understand the detection and mixing process at about $100 \mu \mathrm{m}(3000 \mathrm{GHz})$.

New forms of submicron structures specially designed for the $100-300 \mu \mathrm{m}$ range have recently been produced by the University of Virginia, USA. The cover illustration shows a cross-section through this new type of Schottky barrier diode with its honeycomb structure contacted by a whisker. The actual design was determined by the smallest size of the Schottky contact that can be realized in the laboratory $(0.5 \mu \mathrm{m}$ diameter). The diode typically has a series resistance $R_{\mathrm{s}}$ of $\approx 30 \Omega$ and a capacitance $C_{j}$ of $5 \times 10^{-16} \mathrm{~F}$ leading to a cutoff frequency $\left(2 \pi R_{\mathrm{s}} C_{\mathrm{j}}\right)^{-1}$ of $10 \mathrm{THz}$ which implies that these devices are not only very efficient mixers but also extremely fast video detectors (time constant $\tau \approx 10^{-13} \mathrm{sec}$ ) with a theoretical sensitivity of about $10^{-10} \mathrm{~W} / \sqrt{ } \mathrm{Hz}$ when operated at room temperature.

\section{Quasi-optical mixer}

In contrast to optically coupled devices, the sensitive detector area on a Schottky diode is orders of magnitude smaller than the submillimetre and FIR wavelengths. Antenna structures have therefore to be used to increase the effective detector area. A heterodyne receiver requires at the same time spatial overlap between the signal and the local oscillator.

Waveguide techniques provide efficient coupling in the microwave range. At sub-mm wavelengths below $500 \mu \mathrm{m}$ a different approach must be used owing to to the small physical dimen- 
sions and the increasing importance of surface resistance losses and the skin effect.

A quasi-optical mixer structure (Fig. 3 ) introduced by our group has turned out to be appropriate in the $100-500$ $\mu \mathrm{m}$ range. A whisker that provides contact to the diode acts as a long-wire antenna (several wavelengths in length) with an antenna pattern which is symmetric about the whisker. The coupling efficiency is increased by more than a factor of 10 by placing a corner reflector behind the wire, resulting in a more or-less symmetric antenna beam in the $\mathrm{E}$ - and $\mathrm{H}$-plane and suppressed sidelobes. The open-structure arrangement can be tuned in resonance with the the incoming signal, the $\mathrm{LO}$ radiation frequency and the resulting intermediate frequency (IF) by adjusting the whisker length and the distance between the apex of the corner reflector and the whisker. An extensive evaluation of several types of corner reflectors and corner cubes has turned the fairly simple detector configuration into a widely used antenna coupling structure.

\section{Optically pumped gas laser}

The local oscillator for the mixing process must satisfy numerous requirements that can only be met at the present time by optically pumped gas lasers. The main requirements can be summarized as follows:

- To operate the diode at the optimum working point it is necessary to keep the laser power in the $\mathrm{mW}$ range.

- If it is intended to achieve a spectral resolution $\Delta v / v \approx 10^{-7}$ then the spectral purity must be below this value.

- The observing time is usually at least one hour. The amplitude should remain stable to $\pm 1 \%$ within this period, and the frequency to within $\pm 100 \mathrm{kHz}$. This is a difficult task because temperature changes of $\pm 30 \mathrm{~K}$ are possible when using the KAO.

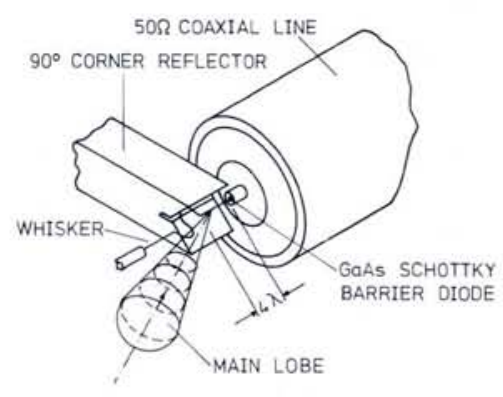

Fig. 3 - Schottky diode in an open structure mixer mount. Matching between the main lobe of the focused signal, the local oscillator beam and the diode detector is achieved by adjusting the corner reflector and the long-wire antenna that also acts as the diode contact.

- A large number of laser lines are required in order to cover the the whole wavelength range. This is because lasers are not easily tunable and the microwave components can only handle an intermediate frequency of $\pm 40 \mathrm{kHz}$. At the moment there are a few hundred laser lines which fulfill these conditions. - The optically pumped gas laser must satisfy the specifications for airborne operation.

Starting with a bulky prototype weighing $1000 \mathrm{~kg}$ we have developed a compact laser system of only $50 \mathrm{~kg}$ in weight that works in any orientation, even when subjected to a linear acceleration of $5 \mathrm{~g}$. Copies are now manufactured by industry and used for other applications.

\section{Acousto-optic analyser}

After mixing and amplifying the signal, an acousto-optic spectrometer (AOS) analyses the incoming spectrum. Based on the Bragg scattering of a coherent light beam at a phase grating, the AOS consists of four components: a $\mathrm{HeNe}$ or diode laser, beam expanding optics, a deflector crystal and a photo detector array (see Fig. 4).

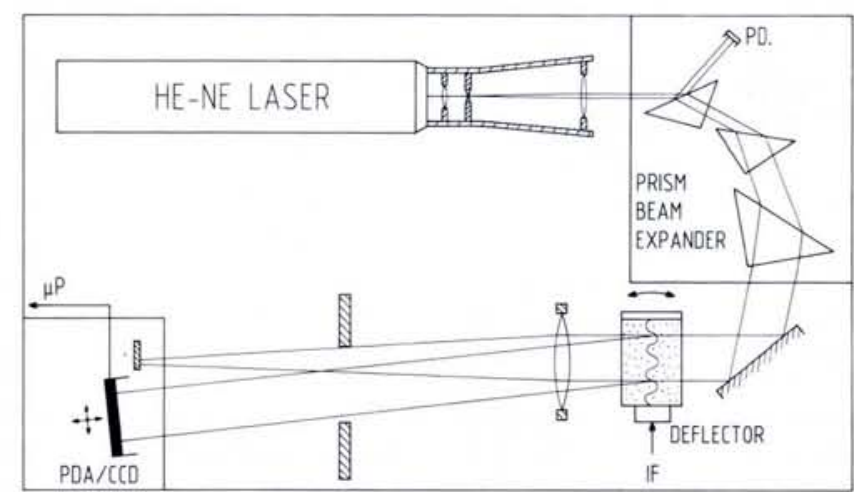

Fig. 4 - Optical layout of the acousto-optic spectrometer. The intermediate frequency signal (IF) in the deflector crystal acts as a phase grating such that light from the HeNe laser is diffracted by the the crystal's density wave and detected by a photodiode array (PDA) or a charge coupled device (CCD) with 1024 or 2048 detector elements.
The amplified IF signal generates a density wave in the deflector crystal in a similar manner to a phase grating (the grating corresponds to the frequency distribution of the signal). A beam from the $\mathrm{HeNe}$ laser is diffracted in the density wave and the distribution of light over different deflection angles is proportional to the frequency of the signal's power distribution. A lens system is used to focus the first-order diffracted light onto a photodiode array or charge coupled device with either 1024 or 2048 detector elements whose outputs are digitized and fed to a personal computer, either directly or via a digital integrator.

The two main design parameters, bandwidth and resolution, determine the choice of material for the Bragg cell. $\mathrm{TeO}_{2}$ is useful for a bandwidth of 50 $\mathrm{MHz} ; \mathrm{LiNbO}_{3}$ is more useful for a bandwidth of $1000 \mathrm{MHz}$ with 1024 channels and a resolution of $\approx 1 \mathrm{MHz} /$ channel. For airborne application, we have developed a compact AOS with a self-supporting structure measuring $50 \times 10 \times$ $20 \mathrm{~cm}$ and weighing $10 \mathrm{~kg}$. Capable of covering a bandwidth of $1000 \mathrm{MHz}$ using 1024 channels, we have used it successfully aboard the KAO since 1988.

\section{Performance}

By combining the components described above it is possible to use only one heterodyne system to span the frequency range from 100 to $500 \mu \mathrm{m}$ at a selected resolution $\Delta v / v$ from $10^{-4}$ to $10^{-8}$ and, if necessary, even two orders of magnitude higher. The heterodyne sensitivity is about $3 \times 10^{-19} \mathrm{~W} / \mathrm{Hz}$ (see Fig. 5) equivalent to about $10^{-16} \mathrm{~W} / \sqrt{ } \mathrm{Hz}$ for a resolution of $1 \mathrm{MHz} /$ channel. The sensitivity range is about a factor of 200 above the quantum noise limit. What is really remarkable is that this performance is achieved even when the complete spectrometer operates at room temperature.

A fundamental and important difference between a microwave spectrum analyzer, or a grating or Fabry-Perot spectrometer, and our heterodyne spectrometer is that the latter is not a scanning device. Instead, all 1024 photodiodes, each with a bandwidth of 1 $\mathrm{MHz}$ are integrated simultaneousiy. The result is a much shorter measuring time and the ability to detect very weak signals. The principle disadvantages with respect to conventional spectrometers are the limited bandwidth of a few $\mathrm{GHz}$ $(\approx \pm 0.1 \%$ of the spectral range) and the need to tune $\pm 20 \mathrm{GHz}$ around the local oscillator frequency. 


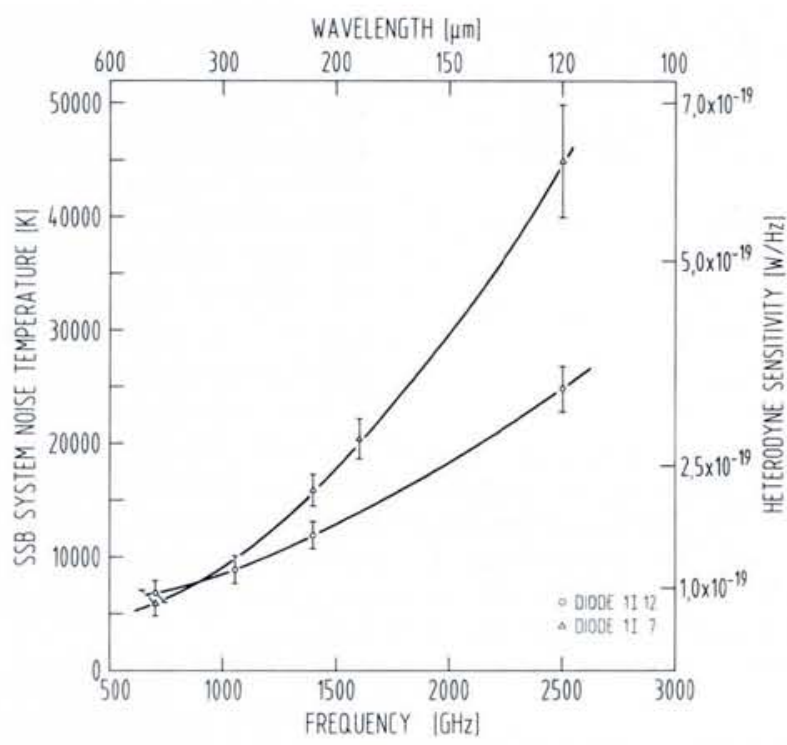

Fig. 5 - Sensitivity of a sub-mm/FIR heterodyne spectrometer for two different GaAs Schottky diodes.

\section{Astrophysical Observations Aboard The KAO}

High $J$ observations of $\mathrm{CO}$ were performed aboard the KAO in September 1988 and from February to March in 1989 using the heterodyne spectrometer. We made the first high resolution detection of $\mathrm{CO}(\mathrm{J}=9-8)$ at $289 \mu \mathrm{m}$, CO $(\mathrm{J}=11-10)$ at $237 \mu \mathrm{m}, \mathrm{CO}(\mathrm{J}=$ $12-11)$ at $217 \mu \mathrm{m}$ and $\mathrm{CO}(\mathrm{J}=14-13)$ at 186 um and studied the the Orion Molecular Cloud 1 (OMC-1) over several arc-minutes around the position IRc2.

Earlier mapping of OMC-1 using the CO $(J=7-6)$ transition aboard the $\mathrm{KAO}$ in 1985 and 1986 showed that the warm gas component extended over several arc-minutes. This is remarkable because the excitation energy required to populate the $J=7$ level is equivalent to a temperature of about $155 \mathrm{~K}$. It then turned out that higher rotational lines could be seen over several arcminutes at high antenna temperatures $\left(T_{\mathrm{A}} \approx 70 \mathrm{~K}\right)$ even though these lines require considerable excitation energies (CO $J=12$ has an excitation temperature of $430 \mathrm{~K}$ ).

We also found that the high- $J$ transitions of $\mathrm{CO}$ in several areas displayed very narrow linewidths equivalent to about $2 \mathrm{~km} / \mathrm{s}$ (Fig. 6); this was not the case for the lower transitions. The high$J$ linewidths at these positions also decreased systematically with an increasing excitation temperature. This was unexpected as line profiles should be wider for the more turbulent, hotter gas located deep inside the cloud as compared with the cold gas in the foreground. A possible explanation may be that the density of carbon dioxide molecules is so high that one only observes a thin skin of hot gas.
We have also investigated the $\mathrm{BN} / \mathrm{KL}$ (Becklin Neugebar/Kleinman Low) outflow close to OMC-1 as well as the continuum emission from dust condensed in this region. Our high- $J$ observations invariably produced some spectra with indications of line wings (Fig. 7) for lines that were broader than those with the usual Gaussian profile. Gas is streaming outwards with velocities of approximately $\pm 50 \mathrm{~km} / \mathrm{s}$. Detailed mapping around one of the regions of high velocity at high spectral resolution using the IRAM 30 metre telescope for $\mathrm{CO}(\mathrm{J}=$ $2-1$ ) indicated that the gas in fact flows rapidly outwards in two opposing

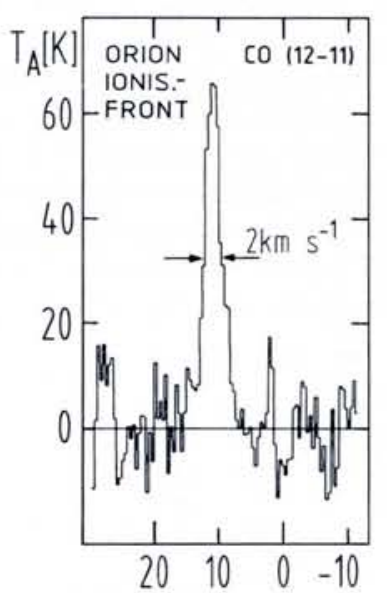

Fig. $6-\mathrm{CO}(\mathrm{J}=12-11)$ emission at the high temperature ionization front in Orion. The antenna temperature $T_{A}$ is plotted as a function of the variation in gas velocity. An extremely narrow spectral line corresponding to a velocity difference of $2 \mathrm{~km} / \mathrm{s}$ is observed for this high-J transition. The narrow width indicates little apparent turbulence in the hot core; lower level transitions from the colder, seemingly more turbulent outer regions are much broader.

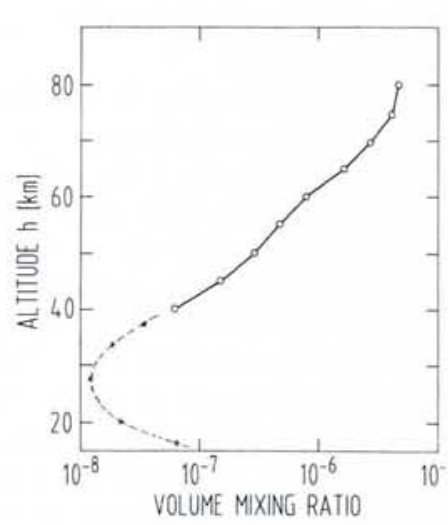

Fig. 8 - Volume mixing of $\mathrm{CO}$ in the Earth's atmosphere measured using sub-mm/FIR heterodyne spectrometry. The dashed curve represents data obtained from a balloon by gas chromatography; the solid curve, results made with an instrument located on the ground.

streams, thus confirming the presence of a new outflow source.

\section{Atmospheric Physics}

Most of the atoms and molecules which are relevant for astronomy are also of interest in atmospheric physics, e.g. $\mathrm{H}_{2} \mathrm{O}, \mathrm{O}_{3}, \mathrm{NH}_{3}, \mathrm{CO}$ and their isotopes. In astronomy, unlike atmospheric physics, the atmosphere is a hindrance - an unavoidable filter with its own emission and adsorption spectra which can falsify interstellar spectra. Our heterodyne spectrometer has been used extensively both on the ground and on the $\mathrm{KAO}$ to investigate the atmospheric transmission curve by measuring adsorption spectra of e.g. $\mathrm{CO}$ and $\mathrm{O}_{3}$.

The profile along a line of sight through the atmosphere, either vertically or horizontally, gives integrated information about the molecular concentration as a function of altitude or position when allowances are made for the Doppler effect and pressure broade-

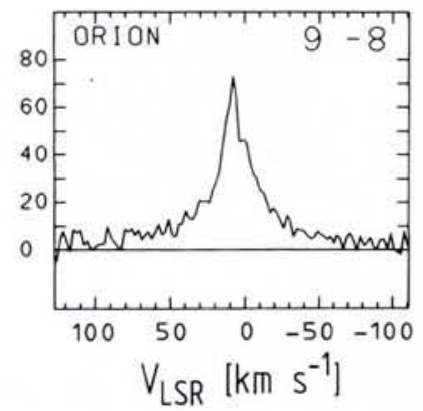

Fig. 7 - Typical spectra of $\mathrm{CO}(\mathrm{J}=9-8)$ close to the $B N / K L$ outflow source in Orion. The broad, non-Gaussian distribution of the antenna temperature (in degrees $K$ ) is thought to arise from two opposing gas streams, each with a velocity $V$ of about $50 \mathrm{~km} / \mathrm{s}$. 
ning. Knowing the temperature and pressure profiles for the Earth's atmosphere it is possible to derive from an accurately measured line profile the volume mixing ratio. Several research groups worldwide are presently using the heterodyne technique to monitor the Earth's atmosphere from airborne platforms. Fig. 8 shows an example of such a profile for $\mathrm{CO}$ obtained by measuring on the ground the $\mathrm{CO}(\mathrm{J}=6-5)$ transition at $434 \mu \mathrm{m}$.

The great advantage of this method is remote observation. It is unnecessary to be at the target position which may be difficult to reach or undesirable owing to safety aspects. Examples include analyses of exhaust gases from the chimneys of power stations and from jet engines, of tokamak plasmas, of toxic gas clouds, etc.

\section{Plasma Physics}

The ion temperature of a tokamak plasma can be measured if one replaces the input signal in Fig. 2 by strong, pulsed laser radiation that has experienced collective Thomson scattering from density fluctuations at the thermal level. Owing to the extremely small Thomson scattering cross-section of $6.66 \times 10^{-29} \mathrm{~m}^{2}$, the signal levels that must be analysed are very low

\section{Some Important and Best-Selling Books from World Scientific}

\section{RESONANCES}

A Volume in Honor of the 70th Birthday of Nicolaas Bloembergen

Cambridge, USA 27 May 1990

eds. M D Levenson (IBM), E Mazur (Harvard), Y R Shen (UC, Berkeley) \& P S Pershan (Harvard)

This volume contains a historical record of one of the great scientists of the 20th century, including a complete bibliography of his papers, four of his most significant publications and evidence of his impact on generations of scientists.

512pp 981-02-0377-2 US\$78 $£ 52$ Pub. date: Nov. 1990 981-02-0378-0(pbk) US $\$ 38$ £25

Proceedings of Nobel Symposium 79

THE BIRTH AND EARLY EVOLUTION OF OUR UNIVERSE

Gräftåvallen, Sweden $11-16$ June 1990

eds. B Gustafsson (Uppsala Univ.), J S Nilsson \& B-S Skagerstam (Chalmers Univ. Tech.)

450pp (approx.) 981-02-0357-8 US\$78 $\$ 52$ Pub. date: Feb 1991 981-02-0358-6(pbk) US\$32 £21 Co-published with Physica Scripta, The Royal Swedish Academy of Sciences.

\section{KNOTS AND PHYSICS}

by Louis H Kauffman (Univ. of Illinois at Chicago)

500pp (approx.) 981-02-0343-8 US\$54 £36 Pub, date: Feb 1991 981-02-0344-6(pbk) US\$28 £19
THE OSKAR KLEIN MEMORIAL LECTURES

Vol. 1: Lectures by $\mathrm{C} \mathrm{N}$ Yang \& S Weinberg with Translated Reprints by $O$ Klein

ed. G Ekspong (Stockholm Univ.)

Volume 1 contains the 1988 lectures on "Symmetry and Physics" and "From the Bethe-Hulthen Hypothesis to the Yang-Baxter Equation", by C N Yang as well as the 1989 lectures on "Beyond the Standard Models" and on "Precision Tests of Quantum Mechanics" by Steven Weinberg. Also included are translations of some of Klein's original papers, and a scientific biography of Klein.

220pp (approx.) 981-02-0352-7 US\$32 \&21 Pub. date: Feb 1991 981-02-0353-5(pbk) US\$16 ع11

\section{PHYSICAL PROPERTIES OF HIGH} TEMPERATURE SUPERCONDUCTORS I

ed. D M Ginsberg (Univ. Illinois, Urbana-Champaign) 528pp 9971-50-683-1 US\$84 256 Pub. date: Mar 1989 9971-50-894-X(pbk) US\$44 £30

\section{PHYSICAL PROPERTIES OF HIGH} TEMPERATURE SUPERCONDUCTORS II

ed. D M Ginsberg (Univ. Illinois, Urbana-Champaign) 712pp 981-02-0124-9 US\$78 $£ 52$ Pub. date: Feb 1990 981-02-0190-7(pbk) US\$38 £25
If. World Scientific

An International Publisher

73. Lynton Mead, Totteridge, London N20 8DH, ENGLAND Telefax: (81) 4463356 Tel: (81) 4462461 Singapore • New Jersey • London • Hong Kong

\section{Optically Pumped FIR Laser Systems from MAN Technology}

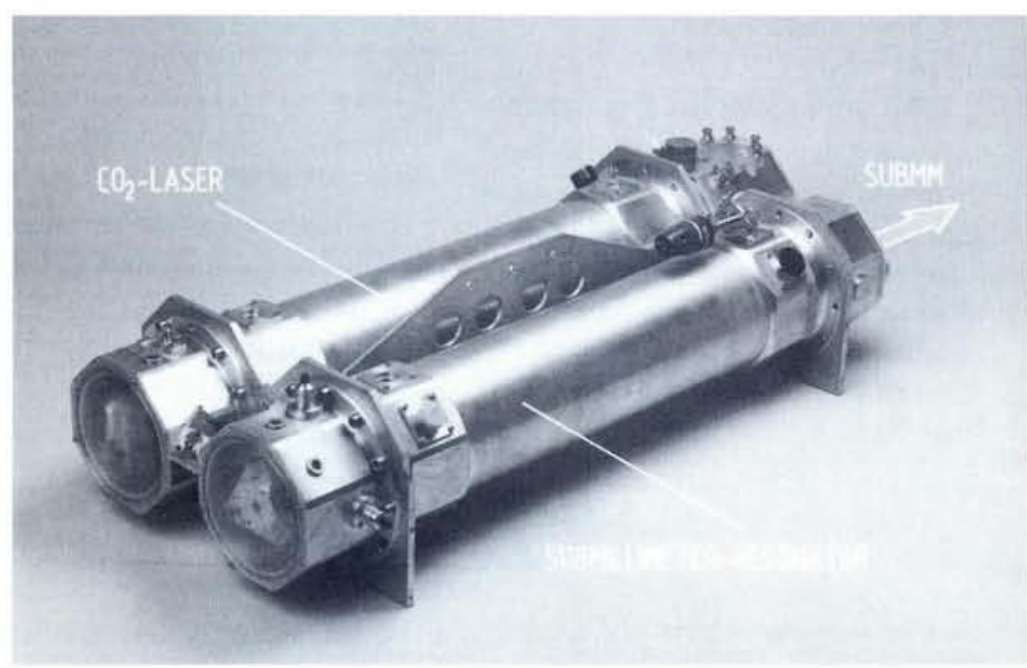

Originally developed as a local oscillator in an airborne FIR heterodyne receiver, our systems have the special integrating structure that make them really meet the stability requirements. No more need for heavy optical benches!

Next to our standard version we can deliver custom defined versions. We have the convincing solution to your requirements, just challenge us!
Applications:

- molecular physics

- solid state physics

- plasma diagnostics

- atmospheric physics

- astronomy

- radar modelling

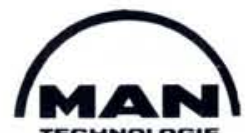

MAN Technologie AG Systemelektronik Marketing, Vertrieb

Dachauer Straße 667

D-8000 München 50

Telefon 0 81 31/9 03-124

Telefax $08131 / 903-220$

Telex $523211-21 \mathrm{mad}$ 


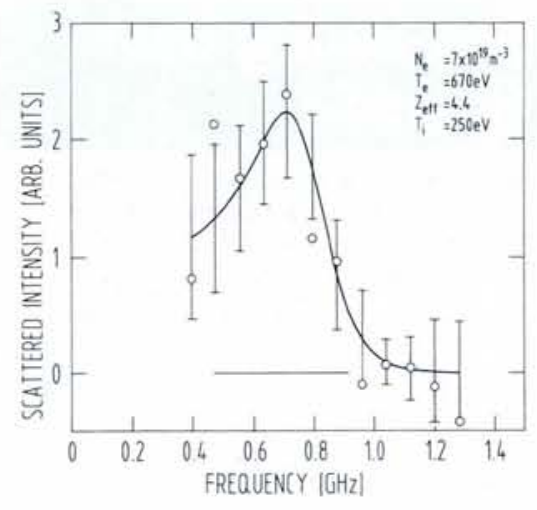

Fig. 9-Measured scattering spectrum for a He plasma with an electron density $N_{e}$ of $7 \times 10^{19} \mathrm{~m}^{-3}$ recorded using a single laser pulse.

$\left(<10^{-18} \mathrm{~W} / \mathrm{Hz}\right)$. The heterodyne technique however, offers several unique opportunities:

- Sensitivity close to the quantum noise limit.

- Rapid detection for laser pulses lasting about 1 us.

- Insensitivity to the noisy tokamak environment.

Successful experiments have recently been carried out by research groups at the Ecole Polytechnique Fédérale in Lausanne and at the University of Düsseldorf using a pulsed $\mathrm{D}_{2} \mathrm{O}$ laser at $385 \mu \mathrm{m}$. A single laser shot was found to be sufficient to determine the ion temperature in $\mathrm{H}, \mathrm{D}$ and $\mathrm{He}$ plasmas with electron densities $N_{e}$ above $5 \times$ $10^{19} \mathrm{~m}^{-3}$ (Fig. 9).

\section{Conclusions}

Since its introduction in astronomy, the sub-millimetre/far infrared heterodyne spectrometer has come to be widely used in other, very different, fields of research. A feature that allowed this extension was the demonstration of an airborne system that had proved itself in a hostile environment. Expected improvements in sensitivity, bandwidth and reliability combined with the capacity to operate at wavelengths smaller than $100 \mu \mathrm{m}$ ensure an exciting future.

\section{FURTHER READING}

Astronomy:

Schmid-Burgk J. et al., Astron. and Astrophys. 215 (1989) 150.

Röser H.P. and Schmid-Burgk J., Sterne und Weltraum 28 (1989) 648

Atmospheric Physics:

Wattenbach R. et al., J. Geophys. Res. 89 , No. D5 (1984) 7285.

Plasma Physics:

Behn R. et al., Phys. Rev. Lett. 62 (1989) 2833.

Sub-mm/FIR techniques:

Chantry G.W., Long-Wave Optics, Vols 1 and 2 (Academic Press, London) 1984.

\section{Nobel Prize in Physics}

The 1990 Nobel Prize in Physics has been awarded jointly to Professors Jerome Friedman and Henry Kendall, both of the Massachusetts Institute of Technology, Cambridge, MA, USA, and Richard Taylor of Stanford University, Stanford, CA, USA for their pioneering investigations of deep inelastic scattering of electrons on protons and bound neutrons which have been of essential importance for the development of the quark model in particle physics.

The three prizewinners were key members of the SLAC-MIT team which confirmed in 1968 clear signs that there exists an inner structure in the proton and neutron of the atomic nucleus. By 1972, interpretation of their results in terms of quarks was assured and work on neutrino scattering started to provide supporting evidence. Their findings therefore paved the way towards today's understanding of the constituents of matter.

An appreciation will be published next month.

\section{Acusto-Optical Spectrometers with Proven Stability}

Elson Research Inc. designs and manufactures acusto-optical spectrum analyzers (AOS) specifically for research applications in radio astronomy or atmospheric physics.

A unique active frequency stabilization technique has earned Elson AOS analyzers a reputation for high sensitivity and excellent reliability.

Each Elson AOS analyzer is tailormade for your specific application; bandwidths available range from 40 $\mathrm{MHz}$ to $1.5 \mathrm{GHz}$, with resolutions from $50 \mathrm{kHz}$ to $10 \mathrm{MHz}$. A subsystem comprises:

* Acusto-optical processor

* Electronics unit

* RF unit

* All power supplies and controls

A subsystem can actually combine up to four spectrometers in a compact chassis for a standard 19" racka rugged, space-saving design which allows easy transportation, installation and maintenance.

When compared to a 1024-channel filter bank for atmospheric research, an Elson AOS offers both wide and narrow resolutions for faster analysis, avoids the need for multiplexing and does not suffer from defective channels. It is also significantly cheaper to purchase and maintain.

The world's leading supplier of commercial AOS analyzers since 1985, Elson Research's recent installations include: The James Clerk Maxwell Telescope in Hawaii (1988), the University of Helsinki (1989) and, for atmospheric physics, the Helsinki University of Technology (double system, 1990).

Elson Research arranges orientation courses, user-training, service-training and maintenance world wide. And ongoing research allows your subsystem to be continually upgraded to the stateof-the-art level.

For more information, please mail or fax Dr. Lauri Malkamäki at:

Elson Research Inc.

P.O. Box 167

Salem, NH 03079-9998

Fax: 603-893-2131

\section{ELSON}

I am interested in reliable AOS analyzers, please send me the following information:

Elson AOS-series specifications

Theory and principles of AOS operation (ISBN 952-90-1704-9)

Application: $\square$ Astronomy $\square$ Atmospheric Physics $\square$ Other

Name and title:

Institute:

Address: 